\title{
Cross-Sectional Shape of Rat Mesenteric Arterioles at Branching Studied by Confocal Laser Microscopy*
}

\begin{abstract}
Atushi NAKANO ${ }^{* *}$, Motomu MINAMIYAMA*** and Hideyuki NIIMI******
This study was aimed to investigate the cross-sectional shape of mesenteric arterioles at branching, using confocal laser microscopy. Wistar rats ( 8 weeks, male) were anesthetized with thiobutabarbital sodium. Blood flow and microvascular network in the mesentery were observed using video microscopy. The rat intestine with mesentery was extracted and the intestinal vasculature was perfused with Krebs-Ringer and then fixed with paraformaldehyde under a static pressure of $100 \mathrm{mmHg}$. A section of mesentery was isolated from the intestine, and spread up to the in vivo geometry based on the intravital microscopic observation. The mesentery section was stained with tetramethyl rhodamine isothiocyanate (TRITC)-phalloidin. The samples were observed under a confocal laser microscope. The cross-sectional image was re-sliced to measure the cross-sectional area and major/minor axes of the best fitting ellipse. The aspect ratio was defined in terms of the minor/major diameter ratio. The extended focus image of mesenteric arterioles showed that the cross-sectional shape was not circular but elliptic-like. The cross-sectional area of the parent vessel decreased from proximal to distal positions. The mean aspect ratio of the parent vessel was approximately 0.5 , while that of the branching vessel was approximately 0.8 . The flattened shape and variation of the cross-sectional area of arterioles requires some correction of in vivo data of the two-dimensional mesenteric microvasculature obtained using intravital microscopy.
\end{abstract}

Key Words: Arteriole, Branching, Confocal Laser Scanning Microscopy, Cross-Section, 3D Reconstruction, Mesentery, Microcirculation

\section{Introduction}

Blood flow in the microvasculature is influenced by the geometry (diameter, length, cross-sectional shape and so on) of single microvessels as well as the topology of microvascular network, including the three-dimensional (3D) structure of branching ${ }^{(1)-(6)}$. Up to now, a number of intravital microscopic studies have been made to measure hemodynamic parameters, such as blood flow velocity and vascular diameter, in the microvasculature in various organs and tissue. In particular, the mesentery has

* Received 31st May, 2005 (No. 05-4054)

** Department of Vascular Physiology, National Cardiovascular Center Research Institute, Suita, Osaka 565-8565, Japan. E-mail: nakano@ri.ncvc.go.jp

*** Department of Clinical Engineering, Hiroshima International University, Kurose, Kurose, Higashi-Hiroshima 724-0695, Japan

**** Tasly Microcirculation Research Center, Peking University Health Science Center, Haidian, Beijing 100083, China been most available for obtaining rheological relationships between blood flow and vascular diameter in the microcirculation $^{(7)-(12)}$.

Intravital microscopy provides the two-dimensional (2D) image of the microvascular network ${ }^{(13)}$, but it is very difficult to obtain cross-sectional informations of microvessels. Recently, confocal laser scanning microscopy has been developed to obtain the 3D images of various cells in microvascular wall ${ }^{(14)-(19)}$. However, there are few investigations on the 3D morphological structure of microvascular cross-section. In a previous report, we studied the 3D structure of rat mesenteric arterioles by using confocal Laser microscopy ${ }^{(19),(20)}$. In this study, we reexamined the 3D confocal laser microscopic images of mesenteric arterioles to make quantitative analysis of the cross-sectional shape and variation of the cross-sectional area of arterioles at branching in rat mesentery.

\section{Materials and Methods}

\subsection{In vivo observation}

Wistar rats (8 weeks, male) were used for mesen- 
teric preparation. The rats $(n=10)$ were anesthetized with thiobutabarbital sodium $(100 \mathrm{mg} / \mathrm{kg}$ b.w.) intraperitoneally, and allowed to respire spontaneously. A carotid artery was cannulated to monitor blood pressure during the experiment. The intestinal mesentery was exteriorized from an abdominal incision and placed into a bath, which was perfused with Krebs-Ringer's solution (KRS) $\left(37^{\circ} \mathrm{C}\right.$, $\mathrm{pH}$ 7.35), and spread out on an observation window on a microscopic stage. Blood flow and microvascular network in the mesentery were observed under an intravital microscope equipped with a transilluminator. Blood flow direction and the 2D geometry of arterioles at branching were recorded.

\subsection{Morphological examination}

2.2.1 Harvest of the microvasculature The rats were sacrificed after the in vivo observation. The rat intestine with mesentery was extracted, and was kept at approximately $4^{\circ} \mathrm{C}$ during the harvest of the microvasculature.

Under a static perfusion pressure of $100 \mathrm{mmHg}$, the intestinal vasculature was perfused from the superior mesenteric artery, first, with $500 \mathrm{~mL}$ KRS to flush out the blood, and then perfused with $4 \%$ paraformaldehyde (PFA) of $200 \mathrm{~mL}$ to fix the tissue. The effluent was drained from the catheter in the mesenteric vein. We isolated 2 or 3 sections (triangular) of the mesentery from the intestine. The microvasculature was stained just after the harvest, and analyzed within 20 hours as described below.

\subsubsection{Confocal microscopy The mesentery sec-} tion was carefully spread out up to the $2 \mathrm{D}$ in vivo geometry of network. The spread section was pinned on a paraffin sheet in shale filled with KRS, so that the geometry of microvascular network might not be changed. It was immersed in $1 \mu \mathrm{g} / \mathrm{mL}$ tetramethyl rhodamine isothiocyanate (TRITC)-phalloidin KRS for 2-3 minutes to stain the microvasculature $^{(21)}$.

The stained mesentery section was observed under a confocal laser scanning microscope (CLSM). The CLSM consisted of a fixed stage microscope (Olympus BX50WI, Japan), a scanning system (Olympus Fluoview FV300, Japan) and a laser unit (546 nm green helium-neon). An objective lens (x60, N.A.0.9, water-immersion; Olympus LUMPlanFl $60 \mathrm{~W}$, Japan) was used. In the vertical scanning, a piezo positioner was mounted to the objective lens. A scanning image size was $512 \times 512$ pixels with the resolution of $0.3 \times 0.3 \mu \mathrm{m}^{2}$, where the observation area was approximately $150 \times 150 \mu \mathrm{m}^{2}$. By using a piezo-positioner, $100-150$ slices of images were selected with $0.3 \mu \mathrm{m}$ interval (the thickness of observed volume: $30-50 \mu \mathrm{m}$ ).

Based on the obtained confocal slice images, a 3D volume including arteriole was reconstructed with $0.3 \times$ $0.3 \times 0.3 \mu \mathrm{m}^{3}$ voxel, using AVS (Ver.5.0, Advanced Visual Systems, USA). The cross-sectional images of arteriole were re-sliced from the reconstructed volume with $5 \mu \mathrm{m}$ interval to characterize the structure and topology of arte- riolar branching.

\subsection{Image analysis}

2.3.1 Sampling of the cross-section Ten units of branched arterioles were selected out of 10 rat mesentery. One unit had one branching point connecting a daughter (branching) vessel with the parent vessel. Based on the intravital microscopic images, the diameters of parent vessels fell in the range between 10 and $41 \mu \mathrm{m}$ (average: $23.8 \mu \mathrm{m}$ ), while the daughter vessels ranged from 7-21 $\mu \mathrm{m}$ (average: $15.0 \mu \mathrm{m}$ ).

In the sampling, the parent vessel was divided into two portions: upstream (proximal) and downstream (distal) ones. In each vessel, $20-25$ cross-sections were examined at an interval of $5 \mu \mathrm{m}$ along the direction of flow from the branching point. These cross-sectional images were carefully taken perpendicular to the axis of vessel, based on the 3D reconstructed image of vessels (see crosssections in Fig. 3). The total numbers of sampling were 462 and 243 cross-sections in the parent and daughter vessels, respectively.

2.3.2 Image of the cross-section A set of the sliced data of cross-sectional images were separated into 12 bit tagged image file format (TIFF). The TIFF images were post-treated using Photoshop (Ver.6.0.2, Adobe, USA). Using the AVS, we reconstructed 3D volume of arteriole, which can be rotated to any direction and sliced a right-angled cross-section with accuracy less than 5 degrees. The luminal surface of vessel cross-section was traced with the freehand selection tool using the NIH Image software (Ver.1.6.3, National Institute of Health, USA). By applying a best-fitting ellipse to the traced lumen, we determined the major $(2 a)$ and minor diameters $(2 b)$ of the ellipse and the angle of inclination $(\theta)$ relative to the focus plane (Fig. 1) using NIH image measurement command. The best-fitting ellipse was defined by equating the second order central moments of the ellipse to the trace. These semi-manual image processing procedures were done by two persons, and the measurement results

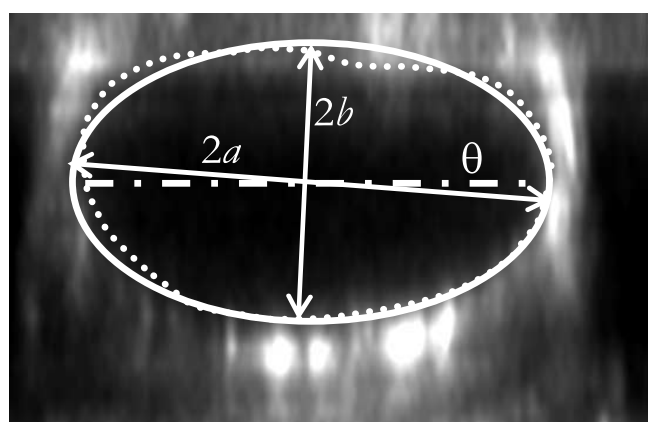

Fig. 1 Tracing (dotted line) of the arteriolar lumen and the best fitting ellipse (solid line). The $a$ and $b$ are the major and minor axes of ellipse, respectively. The $\theta$ is the angle between the major axes and the focal plane (indicated by the dashed line) 
were averaged.

Then, the cross-sectional area $(S)$, aspect ratio $(\alpha)$, and mean diameter $\left(D_{m}\right)$ were calculated as follows:

$$
\begin{aligned}
& S=\pi a b, \\
& \alpha=b / a, \\
& D_{m}=2 \sqrt{a b},
\end{aligned}
$$

where $\alpha$ indicates the degree of elliptic flatness, and $0<$ $\alpha<1$ where $\alpha=1.0$ corresponds to circular shape of the cross-section and $\alpha<1.0$ means collapse of the crosssection.

\subsection{Statistical analysis}

All values are given as mean \pm SD. Appropriate and unpaired Student's $\mathrm{t}$ tests were used to test for statistical significance.

\section{Results}

\subsection{Images of the cross-section at branching}

Figure 2 shows a set of sliced confocal images (upper figure) and the 3D reconstructed image (lower figure) of an arteriole with branching. In the figures, the arteriolar wall was visualized by staining the actin fiber with TRITC-phalloidin.

Figure 3 shows a sequence of the cross-sectional im-
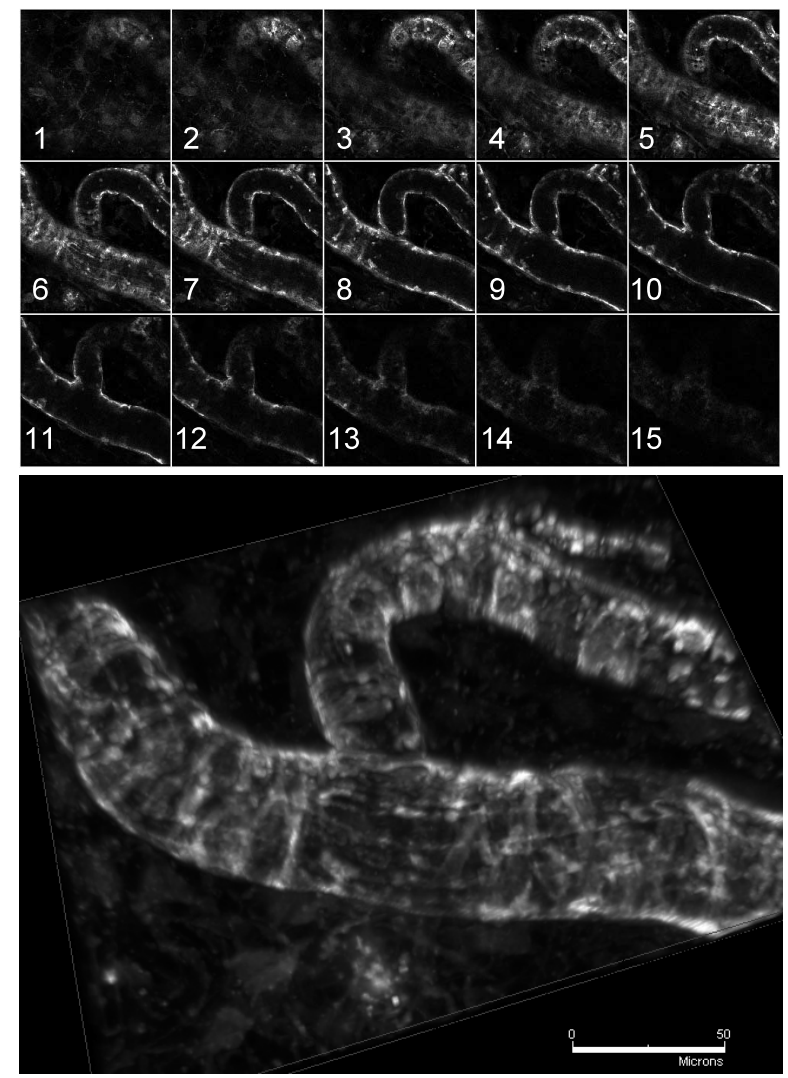

Fig. 2 A set of sliced confocal images (upper figure; 1-15: slice images obtained at each $1.8 \mu \mathrm{m}$ thicknesss) and the 3D reconstructed image (lower figure) of an arteriolar branching in the mesentery ages at intervals of $20 \mu \mathrm{m}$ in the arteriolar branching. In the parent vessel, 7 cross-sections are indicated by -60 , $-40,-20,0,20,40,60$ along the direction of flow from the branching point, while in the branching vessel, 7 cross-sections are indicated by B0, B20, B40, B60, $\mathrm{B} 80, \mathrm{~B} 100, \mathrm{~B} 120$ along the direction of flow from the branching point. Apparently, each cross-sectional image of the parent vessel demonstrates elliptic-like shape, while the cross-sectional shapes in the branching vessel become nearly circular.

\subsection{Change of the cross-sectional parameters around branching}

The cross-sectional area $(S)$ and the aspect ratio $(\alpha)$ of the arteriolar branching shown in Fig. 3 were calculated using Eqs. (1) and (2). Figure 4 shows changes of the cross-sectional area $(S)$ and the aspect ratio $(\alpha)$ from proximal to distal site around the branching. In the parent vessel, the cross-sectional area decreased from approximately $500 \mu \mathrm{m}^{2}$ to $400 \mu \mathrm{m}^{2}$ across the branching point in this exsample. The aspect ratio decreased from approximately 0.5 (proximal) to 0.4 across the branching point, approaching to the proximal level (approximately 0.5 ) at the distal site. In the branching vessel, the cross-sectional area increased from approximately $150 \mu \mathrm{m}^{2}$ at the branching up to $280 \mu \mathrm{m}^{2}$ in the distal site. The aspect ratio increased from approximately 0.6 at the branching, approaching to approximately 0.8 at the distal site.

Apparently, both the cross-sectional area and aspect ratio were influenced markedly by the branching. The area of influence was estimated to be within $40-60 \mu \mathrm{m}$ distance from the branching point in the parent and branching vessel, respectively, which corresponded to 1.5-2.0 times their diameters.

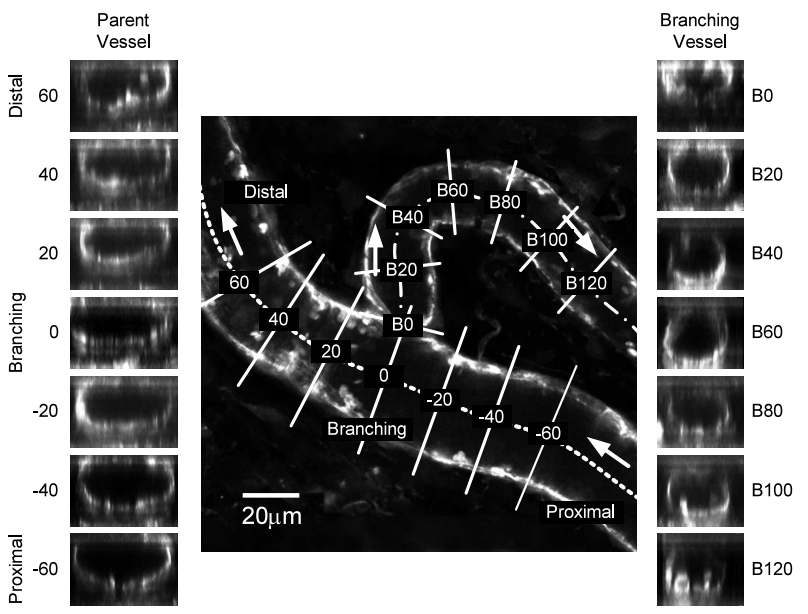

Fig. 3 A sequence of the cross-sectional images at intervals of $20 \mu \mathrm{m}$ taken along the axes of the rat mesenteric arteriole from the branching point. Numbers correspond to the distance $(\mu \mathrm{m})$ from the branching-point in the parent vessel ( -60 to 60 ) and branching vessel (B0 to B120). Arrows indicate the direction of flow in the vessels 

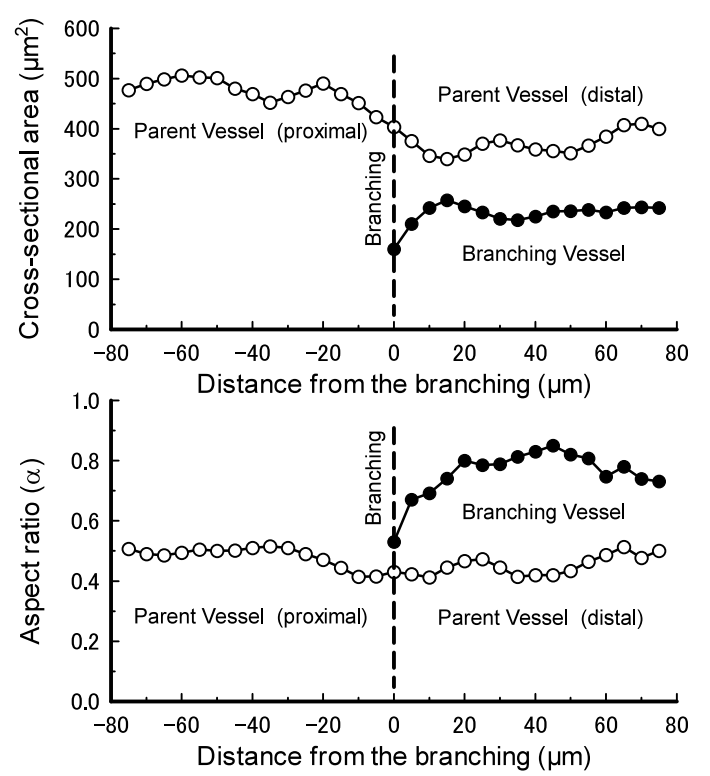

Fig. 4 Changes of the cross- sectional area ( $S$ ) (upper) and the aspect ratio $(\alpha)$ (lower) along the axis of arteriole around branching. Open circle represents a parent vessel, and closed circle shows a branching vessel. The major diameters of parent and branching vessel were approximately $30 \mu \mathrm{m}$ and $20 \mu \mathrm{m}$, respectively

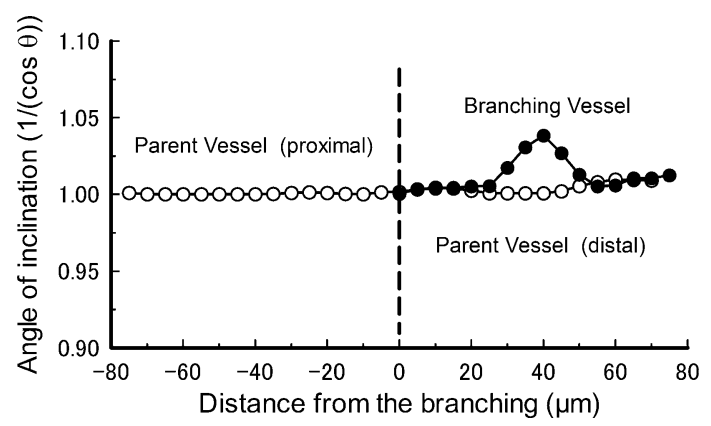

Fig. 5 Changes of the angle of inclination of the best-fitting ellipse relative to the focus plane along the axis of arteriolar branching. Open circle represents a parent vessel, and closed circle shows a branching vessel

It is interesting to note that the aspect ratio of mesenteric arterioles was different between the parent and branching vessels. The aspect ratio of the parent vessel was approximately 0.5 , while the branching vessel was approximately 0.8 . This indicates that the parent vessel was flattened more than the branching vessel.

The angle of inclination $(\theta)$ of the best-fitting ellipse was measured in the arteriole shown in Fig. 3. Figure 5 shows changes of $1 / \cos \theta$ from proximal to distal site around the branching point in the parent and branching vessels. The $1 / \cos \theta$ appeared to remain nearly unity anywhere, indicating that the major axis of the best-fitting ellipse is almost parallel to the plane of focus.

\subsection{A network of arterioles}

To examine cross-sectional change along the microvascular tree, we reconstructed an example of the ar-

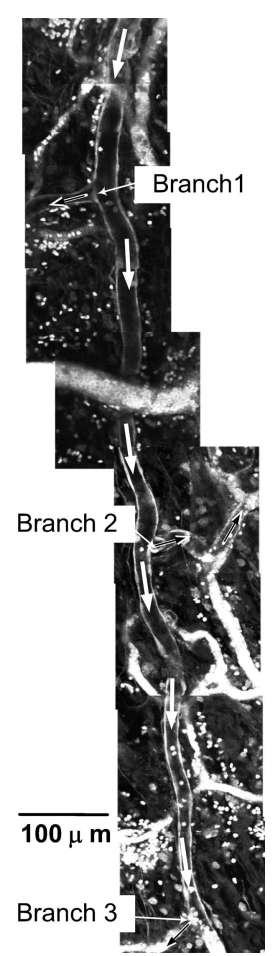

Fig. 6 A network of arterioles in rat mesentery. White arrows indicate the direction of flow in the parent vessel while black arrows indicate the direction of flow in the branching vessels

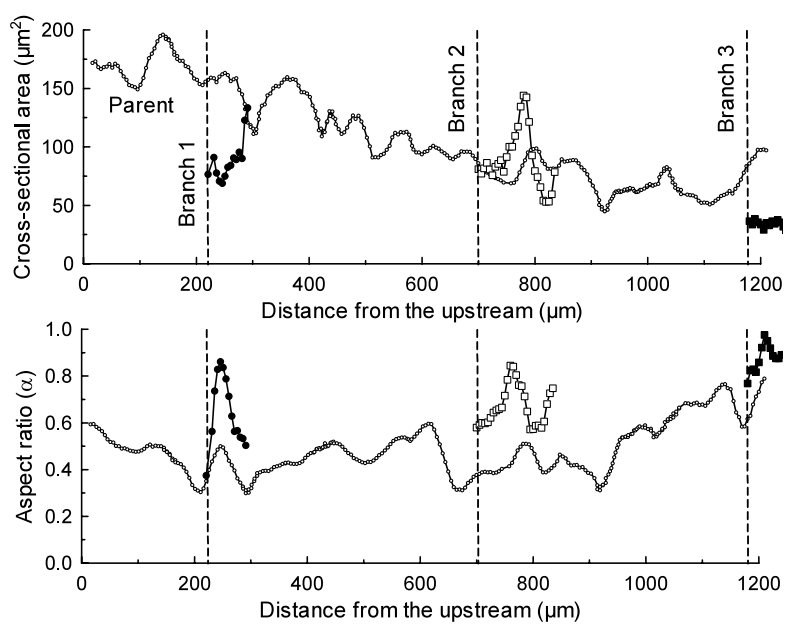

Fig. 7 Changes of the cross-sectional area (upper) and aspect ratio (lower) along the direction of flow in the arteriolar network with three branches. Open circle represents a parent vessel, closed circle: branch 1 vessel, open square: branch 2 vessel and closed square: branch 3 vessel

teriolar network using a sequence of the extended focus images (four segments). Figure 6 shows the montage of the arteriolar network with three branches.

Figure 7 shows changes of the cross-sectional area $(S)$ and aspect ratio $(\alpha)$ along the direction of flow. Apparently, both the cross-sectional area and aspect ratio changed significantly along the flow direction in the parent as well as three branching vessels. In particular, all 


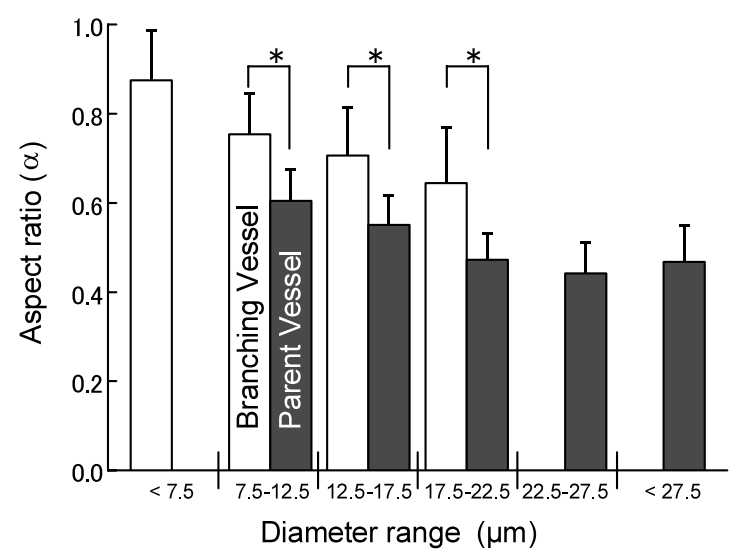

Fig. 8 Relation between the aspect ratio and mean diameter for 462 and 243 cross-sections in parent and branching vessels, respectively in 10 units of branched arterioles out of 10 rats. *significantly different $(P<0.05)$

the branching vessels showed remarkable changes in the aspect ratio, increasing from the branching point and then decreasing at the distal site.

\subsection{Relation between aspect ratio and diameter}

The aspect ratio $(\alpha)$ and mean diameter $\left(D_{m}\right)$ were calculated using Eqs. (2) and (3) for 10 units of branched arterioles (462 and 243 cross-sections in parent and branching vessels, respectively). Figure 8 shows relation between the aspect ratio $(\alpha)$ and mean diameter $\left(D_{m}\right)$ at intervals of $5 \mu \mathrm{m}$ in the parent and branching vessels. In both vessels, the aspect ratio increased with decrease in the mean diameter, but it was larger in the branching vessel, compared to the parent vessel with the same diameter.

\section{Discussion}

Dynamics of blood flow in the microvascular network have been analyzed based on the projected 2D microscopic images of microvessels. In these studies, it has been assumed that the vascular cross-section should be circular with a constant diameter along the axis of vessel $^{(22),(23)}$. Most microvessels in organ and tissue may have complicated and 3D structures, such as bifurcation/confluence, curving or irregular shape of crosssection. Flowing blood cells (red and white) are greatly affected by the 3D morphological structure of vessel. The present study was aimed to make quantitative examination of the cross-sectional shape of branched arterioles in the mesentery using confocal laser microscopy.

The present images (cross-section and extended focus) of a mesentery arteriole around branching have demonstrated that the cross-sectional shape was flattened markedly in the direction of mesenteric membrane (Figs. 2 and 3). Both the cross-sectional area and aspect ratio changed along the axis of vessel from the branching point (Fig.4). Moreover, there appeared marked variations in the cross-sectional shape along the flow direction in an arteriolar tree with three branches (Figs. 6 and 7). These cross-sectional changes of mesenteric arterioles have not been reported except variation in the diameter ${ }^{(22),(23)}$.

There are several factors influencing the shape of the cross-sectional image. One factor is related with the refractive index of tissue, including the arteriolar wall. In the present confocal laser microscopic observation, however, a bath filled with KRS was used together with a waterimmersion objective lens. The refractive index of KRS was almost equal to that of tissue, so that it is not necessary to correct the vertical distance. Moreover, in our preliminary study using Fluoresbrite ${ }^{\circledR}$ YG Microspheres (Polysciences, USA) $(20-40 \mu \mathrm{m}$ diameter $)$, the cross-sections were imaged circular. The difference between the vertical and horizontal diameters of the microsphers was less than 5\%. Therefore, it is reasonable to assume that the refractive effect was negligibly small in the present confocal microscopic observation of the arteriole.

Another influencing factor is related with the arrangement of vessel in the confocal laser microscopic observation. Our samples of mesenteric membrane were carefully placed on a microscopic stage so that the observed arteriole might be parallel to the plane of focus. If the axis of arteriole is completely parallel to the focus plane, the observation should provide the true cross-section. On the other hand, if it is not parallel, the observed cross-section should be flattened in the direction of the membrane thickness. This flatness should be different in the direction from the present observation. Furthermore, our measured angle of inclination was nearly zero to the focus plane (Fig. 5). This indicates that the arteriole should have the major axis of the best-fitting ellipse on the plane of focus. Therefore, it is concluded that our experimental results are not influenced by factors of the arteriolar arrangement.

In our experiment, we used the perfusion fixation technique which has been widely used in vascular morphology and physiology ${ }^{(24)}$. In our preliminary study, we examined changes of the cross-sectional shape of rat mesenteric arterioles at different levels of perfusion pressure $(50,100$ and $180 \mathrm{mmHg})$. No change was observed in the cross-sectional image; The cross-sectional shape of mesenteric arterioles could not be circular even under a high blood pressure of $180 \mathrm{mmHg}$. In our preparation of the mesentery section, the geometry of microvascular network was adjusted to the in vivo $2 \mathrm{D}$ geometry observed under an intravital microscope. The present experiment was focused on the cross-sectional image of arterioles in the mesentery under physiological condition, and thus the mesenteric membrane was not spread (deformed or elongated) more than the in vivo 2D geometry. Such marked deformation (or elongation) may have some influence on the cross-sectional image of arterioles.

Before staining the sample with TRITC-phalloidin, we infused fluorescence substance (FITC-dextran) and observed the cross-section using confocal laser mi- 
croscopy ${ }^{(19)}$. No marked change appeared in the crosssectional shape. It is reasonable to suppose that the present staining might not influence the cross-sectional shape of arteriole.

There are several studies using corrosion casts or angiography of microvasculature in various organs and tissue for examining the 3D arrangement of microvessels ${ }^{(25),(26)}$ or microvascular architecture with cells (smooth muscle cells or pericytes) ${ }^{(27),(28)}$. However, even qualitative analysis on the cross-sectional shape has not been done.

Apparently, the cross-section of mesenteric arterioles appeared of an ellipse-like shape. To consider mechanisms responsible for the elliptic cross-section, let us suppose a single arteriole embedded between two sheets of mesenteric membrane composed of connective tissues etc. ${ }^{(29)}$ The arteriole should be subject to internal and external pressure at the internal and external surface, respectively. The internal pressure should be isotropic in blood, while the external pressure (or normal stress) might be anisotropic in interstitial fluid inside the connective tissues. Presumedly, the normal stress at the mesenteric membrane may be larger than that in the other areas. Owing to the balance of force, the arteriolar cross-section should be deformed from circular to elliptic shape under the existence of different stress levels.

The above mechanism is very plausible for explaining different aspect ratios among arterioles with different diameters. Suppose large and small arterioles embedded between two sheets of mesenteric membrane. It is easily seen that the large arteriole should be subject to external pressure in interstitial fluid between the membranes more than the small arteriole. Accordingly, the large arteriole should be deformed more than the small arteriole, indicating that the aspect ratio of the large arteriole is smaller than that of the small arteriole. In our experiment, the diameter of parent vessels is larger than the branching vessel, and accordingly, the aspect ratio of parent vessels should be smaller than that of branching vessels. This prediction is in agreement with our experimental data in Figs. 4, 7 and 8 to show that the aspect ratios of parent vessels were, on the average, smaller than those of branching vessels.

The present results have shown that the aspect ratios of parent and branching vessels changed along the flow direction from the branching. In particular, change in the aspect ratio of branching vessels was marked from the branching point (Figs. 4 and 7). These features cannot be explained by the above mechanism alone. Both the present cross-sectional areas and aspect ratio of vessel appeared to change within a definite distance from the branching; the influence length fell within 1.5-2.0 times of the diameters of parent and branching vessels. Interestingly, this length was comparable to the entry length of blood flow in arterioles $^{(30)}$. The branching of microvessels is quite singular in the morphology (or geometry) as well as hemodynamics (or hemorheology). In branching, microvascular tethering (or axial) force as well as internal and external pressure may be predominant in the balance of force. For understanding the variation of aspect ratio and crosssectional area in branching, biomechanical analysis will be required under consideration of internal (isotropic) and external (anisotropic) pressure and axial stress.

The present relation between the aspect ratio and the averaged diameter have shown that the aspect ratios of branching vessels were larger, compared to those of parent vessels in the same diameter. Since the aspect ratio of branching vessels changed markedly along the axis of vessel from the bifurcation (Figs. 4 and 7), the branching may be responsible for large aspect ratio of branching vessels, compared with parent vessel.

Up to now, a number of studies using intravital microscopy have been made to measure the diameter of arterioles in the mesentery. Since the intravital microscopy provides the projected 2D image of arterioles alone, the cross-sectional flatness could not be measured even though it was predictable. The present confocal laser microscopy enables us to provide the 3D images of arterioles. Our results using confocal laser microscopy have proved that mesenteric arterioles are not of circular shape but a flattened (elliptic-like) shape in the mesenteric membrane. Furthermore, the cross-sectional area and aspect ratio varies along the axis of arteriole (flow direction). The flattened shape and variation of cross-sectional area of mesenteric arterioles will require some correction of the vascular resistance and related hemodynamic laws established based on in vivo data of mesentery microvasculature obtained using intravital microscopy. These corrections based on the confocal laser microscopic data will be reported elsewhere.

\section{Acknowledgements}

The authors like to thank Drs. J. Seki and M. Masuda for their valuable discussions. The junior author (A.N) thanks Dr. T. Sawamura for his interest in this study.

\section{References}

( 1 ) Pries, A.R. and Secomb, T.W., Rheology of the Microcirculation, Clin. Hemorheol. Microcirc., Vol.29 (2003), pp.143-148.

( 2 ) Ellis, C.G., Wrigley, S.M. and Groom, A.C., Heterogeneity of Red Blood Cell Perfusion in Capillary Networks Supplied by a Single Arteriole in Resting Skeletal Muscle, Circ. Res., Vol.75 (1994), pp.357-368.

( 3 ) Groom, A.C., Ellis, C.G., Wrigley, S.J. and Potte, R.F., Jr., Capillary Network Morphology and Capillary Flow, Int. J. Microcirc. Clin. Exp., Vol.15 (1995), pp.223-230.

( 4 ) Pries, A.R., Secomb, T.W. and Gaehtgens, P., Rela- 
tionship between Structural and Hemodynamic Heterogeneity in Microvascular Networks, Am. J. Physiol., Vol.269 (1995), pp.H1713-1722.

( 5 ) Kalsho, G. and Kassab, G.S., Bifurcation Asymmetry of the Porcine Coronary Vasculature and Its Implications on Coronary Heterogeneity, Am. J. Physiol., Vol.287 (2004), pp.H2493-2500.

( 6 ) Niimi, H., Nakano, A., Koami, Y. and Seki, J., Heterogeneity of Capillary Flow in the Retrograde Microcirculation Induced in Rat Limb by Arteriovenous Shunting, Microvasc. Res., Vol.70 (2005), pp.23-31.

( 7 ) Zweifach, B.W. and Lipowsky, H.H., Quantitative Studies of Microcirculatory Structure and Function III. Microvascular Hemodynamics of Cat Mesentery and Rabbit Omentum, Circ. Res., Vol.41 (1977), pp.380390.

( 8 ) Zweifach, B.W. and Lipowsky, H.H., Pressure-Flow Relations in Blood and Lymph Microcirculation, Handbook of Physiology, Section 2: The Cardiovascular System, Vol.IV, Part 1 Microcirculation, Edited by Renkin, E.M. and Michel, C.C., (1984), pp.251-307, Am. Physiol. Soc., Oxford Press.

(9) Lipowsky, H.H., Usami, S. and Chien, S., In Vivo Measurement of Apparent Viscosity and Microvessel Hematocrit in the Mesentery of the Cat, Microvasc. Res., Vol.19 (1980), pp.297-319.

(10) Minamiyama, M. and Hanai, S., Propagation Properties of Vasomotion at Terminal Arterioles and Precapillaries in the Rabbit Mesentery, Biorheology, Vol.28 (1991), pp.275-286.

(11) Seki, J., Flow Pulsation and Network Structure in Meseteric Microvasculature of Rats, Am. J. Physiol., Vol.266 (1994), pp.H545-553.

(12) Seki, J. and Lipowsky, H., In Vivo and in Vitro Measurements of Red Cell Velocity under Epifluorescence Microscopy, Microvasc. Res., Vol.38 (1989), pp.110124.

(13) Tozer, G.M., Ameer-Beg, S.M., Baker, J., Barber, P.R., Hill, S.A., Hodgkiss, R.J., Locke, R., Prise, V.E., Wilson, I. and Vojnovic, B., Intravital Imaging of Tumour Vascular Networks Using Multi-Photon Fluorescence Microscopy, Adv. Drug Deliv. Rev., Vol.57 (2005), pp.135-152.

(14) He, P., Understanding Vascular Structure and Function by Confocal Imaging of Endothelial cells in vivo, Microscopy Analysis, Vol.39 (1999), pp.9-11.

(15) He, P. and Adamson, R.H., Visualization of Endothelial Cleft and Nuclei in Living Microvessels with Combined Reflectance and Fluorescence Confocal Microscopy, Microcirculation, Vol.2 (1995), pp.267-276.

(16) Arribas, S.M., Daly, C.J. and McGrath, I.C., Measurement of Vascular Remodeling by Confocal Microscope, Method in Enzymology, Vol.307 (1999), pp.246-273, Academic Press.

(17) Itoh, J., Kawai, K., Serizawa, A., Yasumura, K., Ogawa, K. and Osamura, R.Y., A New Approach to Three Dimensional Reconstructed Imaging of Hormone-Secreting Cells and Their Microvessel Environments in Rat Pituitary Glands by Confocal Mi- croscopy, J. Histochem. Cytochem., Vol.48 (2000), pp.569-577.

(18) Jariyapongskul, A., Nakano, A., Yamaguchi, S., Nageswari, K. and Niimi, H., Maturity of Pericytes in Cerebral Neocapillaries Induced by Growth Factors, Clin. Hemorheol. Microcirc., Vol.29 (2003), pp.417421.

(19) Hanai, S., Masuda, M. and Minamiyama, M., Intravital Ovservation of Microvessel Cross-sections Using a Confocal Laser Scanning Microscope (CLSM), Microcirculation Annual, Vol.10 (1994), pp.207-208.

(20) Nakano, A., Minamiyama, M. and Niimi, H., ThreeDimensional Structure of Rat Mesenteric Arterioles at Branching Using Confocal Laser Scanning Microscopy, Microcirculation Annual, Vol.19 (2003), pp.47-48.

(21) Hansen-Smith, F.M., Watson, L., Lu, D.Y. and Goldstein, I., Griffonia Simplicifolia I: Fluorescent Tracer for Microcirculatory Vessels in Nonperfused Thin Muscles and Sectioned Muscle, Microvasc. Res., Vol.36 (1988), pp.199-215.

(22) Kiani, M.F., Cokelet, G.R. and Sarelius, I.H., Effect of Diameter Variability along a Microvessel Segment on Pressure Drop, Microvasc. Res., Vol.45 (1993), pp.219-232.

(23) Pries, A.R., Schonfeld, D., Gaehtgens, P., Kiani, M.F. and Cokelet, G.R., Diameter Variability and Microvascular Flow Resistance, Am. J. Physiol., Vol.272 (1997), pp.H2716-2725.

(24) Tateishi, N., Suzuki, Y., Soutani, M. and Maeda, N., Flow Dynamics of Erythrocytes in Microvessels of Isolated Rabbit Mesentery: Cell-Free Layer, J. Biomech., Vol.27 (1994), pp.1119-1125.

(25) Hashimoto, H., Ishikawa, H. and Kusakabe, M., Three-Dimensional Investigation of Vascular Nets by Fluorochrome-Labeled Angiography, Microvasc. Res., Vol.55 (1998), pp.179-183.

(26) Lametschwandtner, A., Minnich, B., Kachlik, D., Setina, M. and Stingl, J., Three-Dimensional Arrangement of the Vasa Vasorum in Explanted Segments of the Aged Human Great Saphenous Vein: Scanning Electron Microscopy and Three-Dimensional Morphometry of Vascular Corrosion Casts, Anat. Rec. A. Discov. Mol. Cell. Evol. Biol., Vol.281 (2004), pp.1372-1382.

(27) Bhutto, I.A. and Amemiya, T., Microvascular Architecture of the Rat Choroid: Corrosion Cast Study, Anat. Rec., Vol.264 (2001), pp.63-71.

(28) Ikebe, T., Shimada, T., Ina, K., Kitamura, H. and Nakatsuka, K., The Three-Dimensional Architecture of Retinal Blood Vessels in KK Mice, with Special Reference to the Smooth Muscle Cells and Pericytes, J. Electron Microsc., Vol.50 (2001), pp.125-132.

(29) Bloom, W. and Fawcett, D.W., Connective Tissue Proper, A Textbook of Histology (12th Ed), (1994), pp.158-171, Chapman and Hall.

(30) Fung, Y.C., Entry Flow, Bolus Flow and Other Exsamples, Biodynamics: Circulation, (1984), pp.259-264, Springer-Verlag. 